Relations industrielles

Industrial Relations

\title{
Personnel Management, A Situational Approach, by David P. Crane, Belmont, Wadsworth Publishing, 1974, 572 p.
}

Jean Ladouceur

Volume 30, numéro 4, 1975

URI : https://id.erudit.org/iderudit/028672ar

DOI : https://doi.org/10.7202/028672ar

Aller au sommaire du numéro

Éditeur(s)

Département des relations industrielles de l'Université Laval

ISSN

0034-379X (imprimé)

1703-8138 (numérique)

Découvrir la revue

Citer ce compte rendu

Ladouceur, J. (1975). Compte rendu de [Personnel Management, A Situational Approach, by David P. Crane, Belmont, Wadsworth Publishing, 1974, 572 p.] Relations industrielles / Industrial Relations, 30(4), 804-804.

https://doi.org/10.7202/028672ar

Tous droits réservés (C) Département des relations industrielles de l'Université Laval, 1975
Ce document est protégé par la loi sur le droit d'auteur. L'utilisation des services d'Érudit (y compris la reproduction) est assujettie à sa politique d'utilisation que vous pouvez consulter en ligne.

https://apropos.erudit.org/fr/usagers/politique-dutilisation/ 
of the Council. Further, the author makes continual reference to statements of various members of the Council without identifying either the nature of their duties on the Council or their position in either the government or employee organization hierarchy. Accordingly, the volume would have less utility for those who are not familiar with the industrial relations system in the Public Service. However, in view of the dearth of literature on the federal industrial relations environment, the author has made a significant contribution. His work should appeal to specialists and, to a lesser degree, a general audience interested in industrial relations in the Federal Public Service.

\section{A. Gray GILLESPIE}

Staff Relations Officer

Canadian Penitentiary Service

Personnel Management, A Situational

Approach, par David P. Crane, Belmont, Wadsworth Publishing, 1974, $572 \mathrm{p}$.

Voici un livre complet sur la direction du personnel, avec une optique différente. La philosophie de l'auteur reflète l'environnement dynamique des entreprises et des travailleurs, dans lequel on peut difficilement appliquer des principes immuables. En conséquence, le directeur du personnel ou le gérant doit analyser la situation pour y déceler les variables humaines, techniques et organisationnelles susceptibles de solution ner les problèmes de ses employés.

Le premier chapitre de l'ouvrage souligne le rôle significatif de l'administration du personnel dans les organisations modernes, en tenant compte des changements socio-économiques récents. Par la suite, quelques facteurs de la productivité du travailleur sont identifiées; cependant, l'auteur tend à simplifier ce sujet, puisque les travaux de Herzberg et de Sutermeister démontrent la complexité des facteurs dans l'efficacité des employés.

Le troisième chapitre met en évidence l'importance grandissante de politiques et de plans bien articulés pour la gestion des entreprises; plusieurs exemples illustrent adéquatement la nature et les conséquences de ces politiques.

Par la suite, on retrouve les fonctions classiques, telles que la sélection, le placement, le développement et la rémunération du personnel. Trois chapitres sont consacrés aux relations patronales-syrdicales, en mettant l'accent sur l'interaction des facteurs influençant les négociations collectives et les différends de travail. Enfin, le chapitre 20 identifie les changements probables dans l'avenir et souligne les secteurs stratégiques pour améliorer la «qualité de la vie de travail».

Bien que ce livre reprenne les mêmes thèmes que les ouvrages populaires en direction du personnel (v.g. Pigors \& Myers, McFarland, Megginson), il insiste sur l'analyse adéquate des situations concrètes pour diagnostiquer un problème avant de prendre une décision administrative; à cet effet, l'utilisation de problèmes et d'études de cas dans les chapitres vise à développer cette habileté chez le lecteur. Il convient de noter le langage simple de l'auteur, l'utilité d'une soixantaine de tableaux, ainsi que la présentation soignée du livre. Bref, c'est un ouvrage complet pour l'étudiant et pour le praticien qui veut mettre à jour ses connaissances.

\section{Jean LADOUCEUR}

\section{Université de Moncton}

\section{Petit dictionnaire de droit québécois et} canadien, par Me Dominique Pagé, Montréal, Fides, 1975, 167 p.

Cet ouvrage présente les définitions des termes légaux les plus courants dans les domaines juridiques suivants répartis en treize chapitres d'inégale importance: généralités, administration de la justice, droit civil, procédure civile, droit commercial, loi des compagnies d'assurance, droit municipal, droit ouvrier (sic), droit pénal ou criminel, droit constitutionnel et international, loi des faillites et droit aérien.

On comprendra que couvrir un champ aussi vaste en moins de cent cinquante pages est un défi peu ordinaire. On ne sera donc pas surpris de rester sur son appétit devant certaines définitions et de regretter certaines omissions, comme par exemple, injonction. En matière du travail, il n'y a que treize termes. L'auteur parle de "droit ouvrier», de "conflit ouvrier» qui sont des expressions dés:è̀tes. La négociation est bien davantage qu'un «moyen de régler un conflit ouvrier entre employeur et employés 